\title{
Considérations sur l'écologie des estuaires
}

\author{
par J.-M. Pérès \\ Membre de I'Institut \\ Directeur de la Station Marine d'Endoume et du \\ Centre d'Océanographie, Marseille
}

Le temps qui m'est imparti ne peut évidemment me permettre que de donner un aperçu très général des aspects essentiels de l'écologie des estuaires, sujet dont je ne suis pas spécialiste, mais sur lequel existe une bibliographie extrêmement importante.

Tout d'abord, je dirai qu'il faut s'entendre sur le terme même d'estuaire, dont je considère qu'il correspond à une large ouverture sur la mer de la partie terminale du fleuve, c'est-à-dire à une libre circulation des eaux. J'éliminerai donc de mon propos les lagunes côtières, plus ou moins bien séparées de la mer par un cordon littoral, même si ces lagunes reçoivent des apports importants d'eaux continentales.

Les études écologiques étant obligatoirement fondées sur la connaissance des facteurs physiques et chimiques $\mathrm{du}$ milieu qui conditionnent la présence des espèces en fonction de leurs exigences et tolérances propres (autécologie), je donnerai tout d'abord un bref aperçu des caractéristiques des facteurs ambiants en milieu d'estuaire. Bien entendu, je laisserai de côté les problèmes d'ordre microbiologique qui doivent être traités par le Dr Aubert.

Une zone d'estuaire, où s'affrontent les eaux douces et les eaux marines, est caractérisée, du point de vue des facteurs ambiants, par deux faits essentiels :

- l'existence de gradients accusés de ces facteurs;

- l'existence, en un point donné de l'estuaire, d'une instabilité temporelle élevée.

En ce qui concerne les deux facteurs principaux, et dont les valeurs ne dépendent que des modalités du mélange, à savoir la température $\left(T^{\circ}\right)$ et la salinité $(S \%$ ) , je rappellerai simplement que les gradients sont, les uns horizontaux, les autres verticaux. En ce qui concerne les premiers, la $S \%$ moyenne, bien entendu, croît en direction de la mer, en même temps, d'ailleurs, que décroît la charge solide (mais pour ce qui concerne les problèmes de transport et de sédimentation, je laisse la parole à mon collaborateur M. Chamley, qui en traitera dans quelques instants); il $\mathrm{y}$ a presque toujours un gradient horizontal de $T^{\circ}$, l'eau de mer ayant une amplitude de variation annuelle plus faible que les eaux continentales. Le gradient vertical de salinité, croissant de la surface au fond, est toujours net; celui de température l'est généralement moins. La conjonction des gradients verticaux $T^{\circ}$ et $S \%$ engendre évidemment des différences de densité, d'autant plus accusées que $T^{\circ}$ est plus élevée, d'où une tendance importante à la stratification en zone tropicale et, en été, en zone tempérée. Les barrières de densité, qui font de certaines masses d'eau des estuaires de véritables milieux fermés, ont une grande importance écologique.

Je passerai, intentionnellement, sur des aspects mineurs du mélange des eaux douces et des eaux de mer, tels par exemple, de légers changements de la balance des ions, telle qu'elle existe dans les eaux de mer; ces altérations n'ont que peu d'influence sur les êtres vivants.

Avant d'envisager les propriétés chimiques non conservatives, dont les fluctuations sont influencées par les processus biologiques, je rappellerai simplement que les écologistes ont l'habitude un peu simpliste à mon sens, de répartir les estuaires en deux groupes, suivant les modalités de la circulation :

- les estuaires à deux flux (rivière et marées);

- les estuaires à coin salé, dans lesquels le flux d'eau douce domine complètement la circulation.

En ce qui concerne les propriétés non conservatives, j'examinerai brièvement ce qui concerne l'oxygène dissous, les sels nutritifs (azote et phosphore sous forme minérale), les matières organiques, propriétés dont les fluctuations sont d'ailleurs étroitement liées. 
D'une façon générale on peut admettre qu'un fleuve apporte à l'estuaire des eaux qui sont :

- plus pauvres en oxygène dissous que les eaux de mer de surface, lesquelles sont même, le plus souvent, sursaturées en $\mathrm{O}_{2}$;

- riches en matériel organique non vivant, d'origine domestique ou industrielle;

- riches en sels minéraux nutritifs, du fait de la minéralisation d'une partie du matériel organique au sein même des eaux fluviales et du lessivage des engrais dans les zones agricoles du bassin versant.

Au niveau de l'estuaire on assiste aux phénomènes suivants.

A. - Le plancton fluvial (potamoplancton) est tué au contact de l'eau saumâtre ou marine et tend à tomber en direction du fond.

B. - L'apport d'azote et phosphore minéraux stimule la multiplication des espèces phytoplanctoniques marines, d'autant plus que, curieusement, la plupart de celles-ci ont un optimum de salinité de l'ordre de 15-20\%, bien inférieur aux valeurs qu'elles trouvent en mer même; la richesse relative du phytoplancton, et du zooplancton qui s'en nourrit, conduit, surtout dans le cas des estuaires à deux flux, où certaines masses d'eau vont et viennent au rythme des marées (avec parfois en plus des mécanismes de migrations verticales circadiennes, renforçant l'isolement du peuplement), à un accroissement de la quantité de matériel organique parvenant au niveau du fond; de plus, des valeurs anormales du rapport atomique $N / P$ dans certains volumes d'eau hydrologiquement clos, conduisent parfois à des poussées anarchiques de certaines espèces phytoplanctoniques (eaux décolorées) qui, avec des densités qui peuvent atteindre plusieurs dizaines de millions de cellules par litre, réalisent une véritable autopollution. De plus, la pollution thermique contribue, dans les régions tempérées, à créer des conditions analogues à celles qui existent naturellement dans certaines régions tropicales, où les eaux décolorées sont imputables à des Dinoflagellés toxiques pour divers animaux marins et pour l'homme. Cette perturbation physique est de nature à aggraver l'autopollution.

C. - Les phénomènes de précipitation et floculation, dus au mélange d'eau douce et d'eau de mer, tendent également à faire descendre sur le fond une partie des matières organiques détritiques (et des argiles) apportées par le fleuve.

D. - Certaines petites molécules organiques dissoutes (urée, amines, acides aminés, glucides) peuvent être utilisées comme source de carbone, préférentiellement au $\mathrm{CO}_{2}$, par diverses algues planctoniques, mais surtout par des algues benthiques appartenant au groupe des algues vertes et dont la production de grosses molécules organiques, dont une certaine fraction ira également vers le fond, contribue encore à accroître la tendance du milieu estuarien à l'autopollution. Cette tendance peut également être aggravée par la pollution thermique.

De tous les phénomènes qui viennent d'être brièvemént résumés, il résulte qu'une caractéristique assez générale des estuaires est que leur fond reçoit des quantités très importantes de matériel organique dont la dégrada- tion, principalement par des micro-organismes, entraîne une consommation d'oxygène importante. De plus, comme la pycnocline s'oppose aux échanges verticaux, on observe, en définitive, en ce qui concerne l'oxygène dissous, l'existence de deux couches d'eaux souvent très distinctes :

- une couche superficielle, dont la teneur en oxygène dissous dépend des conditions du mélange eau douce/eau de mer et de l'activité physiologique des populations planctoniques;

- une couche profonde, anoxique, d'où toute vie autre que microbienne est bannie. L'anoxie peut aussi n'être que momentanée (et alors souvent saisonnière) si le fleuve présente de grandes irrégularités de débit, le régime de crue ayant pour résultat de «nettoyer»le fond.

De plus, il faut noter que, bien que les eaux côtières bénéficient toujours, en provenance de l'estuaire, d'un certain enrichissement en azote et phosphore minéraux qui contribue à les fertiliser, cet apport ne correspond qu'à une fraction de ce que le fleuve transportait avant de parvenir à l'estuaire. En effet, on a vu qu'une fraction de ces sels minéraux est utilisée, au sein même de l'estuaire, par ses populations végétales propres, mais que la plus grande partie des déchets, issus des population estuariennes, tend à s'accumuler au fond de l'estuaire même; aussi la minéralisation in situ de ce matériel organique ne peut-elle guère profiter aux producteurs primaires et un estuaire peut-il être considéré comme un véritable piège à sels nutritifs, "a nutrient trap », sauf dans le cas signalé précédemment des fleuves à régime très irrégulier.

Encore que l'étude des facteurs ambiants m'ait conduit à faire déjà de larges allusions aux conditions écologiques des estuaires, il convient maintenant de mettre les êtres vivants des milieux d'estuaires au premier plan de l'exposé.

Les peuplements d'estuaire, parmi lesqueks je ne ferai pas figurer les espèces potamotoques ou thalassotoques qui ne font que transiter par l'estuaire, appartiennent au grand ensemble des peuplements d'eaux saumâtres dont ils ne sont qu'un des aspects. En principe, un peuplement saumâtre est constitué de la juxtaposition de trois éléments (dont les proportions respectives varient en fonction des gradients évoqués précédemment) : un élément d'eau douce (dulçaquicole), un élément marin, un élément proprement saumâtre. Ce cadre général est, en fait, assez artificiel et doit être assorti de précisions, dont les principales me paraissent être les suivantes.

A. - Les espèces d'eau douce sont toujours minoritaires, sauf dans les zones où la salinité ne dépasse pas 3 - 5\%; elles sont absentes ou rares dans les zones où elle dépasse $10 \%$.

B. - L'euryhalinité des espèces typiquement marines est très variable suivant les groupes, les espèces, et suivant l'état physiologique, la régulation ionique est dans une certaine mesure liée à la température.

C. - Le nombre de ces espèces typiquement marines existant dans une zone donnée d'un estuaire dépend prineipalement de $S \%$ (valeur moyenne et valeurs extrêmes) et ce nombre diminue en direction du fleuve, mais non de façon régulière : on peut dire assez grossièrement qu'il y a deux discontinuités, l'une vers $25-28 \%$, l'autre vers $10-12 \%$ 
D. - Il existe des formes typiquement saumâtres, qu'on trouve principalement entre $10 \%$ et $25 \%$ environ; elles sont, dans leur immense majorité, d'origine marine; leur statut systématique varie par rapport aux formes marines dont elles sont dérivées : espèce, sous-espèce, race physiologique. Un peuplement d'estuaire (et ceci est vrai des peuplements saumâtres en général) a un caractère local, au moins au niveau de la région (ou de la province) biogéographique; il n'y a pas de faune saumâtre à l'échelon mondial; néanmoins, certaines espèces très tolérantes, celles notamment qui existent dans les milieux portuaires plus ou moins pollués (risques d'introduction d'espèces exogènes par les coques ou les ballasts des navires), peuvent se retrouver dans des estuaires très divers, tout au moins en zone tempérée, moins souvent en zone intertropicale, jamais dans les estuaires arctiques.

E. - Dans le sens du gradient décroissant de salinité, et jusqu'à une valeur limite de 3 à $5 \%$, on observe une diminution assez générale du nombre des espèces représentées; cette diminution de l'indice de diversité est surtout frappante lorsque l'effet d'eutrophisation, au niveau de l'estuaire, est important et conduit à l'exubérance des populations des espèces les plus tolérantes ou les mieux adaptées.

Le problème de l'eutrophisation, au sens strict du terme, c'est-à-dire en concevant qu'il représente une fertilisation qui n'a pas un caractère excessif, est extrêmement important. On doit souligner, en particulier, que l'eutrophisation en milieu d'estuaire ne peut avoir un caractère satisfaisant que dans la mesure où l'instabilité temporelle des facteurs ambiants n'est pas trop importante, tant à très court terme (cycle de marée) par exemple, qu'à moyen terme (écarts saisonniers de débits); les instabilités du deuxième type peuvent, en effet, provoquer des mortalités massives génératrices d'une libération brutale de quantités importantes de matériel organique et, corrélativement, des processus d'autopollution qui donnent alors à l'eutrophisation un caractère épisodiquement excessif. L'eutrophisation excessive permanente, courante dans les lacs ou les lagunes relativement fermées, n'est pas un phénomène courant en estuaire. Il faut souligner aussi que les peuplements d'estuaires présentent, le plus souvent, en raison des gradients verticaux signalés précédemment, une stratification accusée; celle-ci est très importante à considérer, du point de vue de l'art de l'ingénieur, pour les problèmes de salissures des circuits industriels d'eau, pour les problèmes de corrosion (métaux, maçonnerie, bois).

Il me parait hors de propos, devant un auditoire de techniciens, de m'étendre sur la composition du plancton et du benthos des milieux saumâtres. Il n'y a pas grandchose à dire des unités de peuplement au sens écologique du terme. Elles sont difficilement discernables, tout au moins dans le domaine benthique. On ne peut guère, dans les estuaires, reconnaître que des espèces indicatrices d'un ensemble de conditions fluctuant autour d'une certaine moyenne.

Hors les remarques très générales d'ordre écologique que j'ai exposées plus haut, je voudrais terminer par quelques réflexions sur les problèmes de protection du milieu d'estuaire, pour conserver à celui-ci un certain potentiel de production de matière vivante.

Si l'on analyse le peuplement global des estuaires en fonction de la stabilité de sa composition dans une zone donnée, on s'aperçoit qu'on peut y reconnaître trois groupes d'espèces :

a) les espèces permanentes, qui sont celles dont il a été question précédemment et qui présentent une tolérance particulière (ou une adaptation génétique) au milieu estuarien et à ses fluctuations; leur permanence peut d'ailleurs être soumise à des éclipses temporaires lorsque certains facteurs ambiants prennent une intensité extrême, notamment en cas de crue exceptionnelle ou d'étiage anormal;

b) les espèces sporadiques, dont la présence en estuaire correspond également à des conditions exceptionnelles des facteurs ambiants; leur intérêt économique est nul et je n'y reviendrai pas;

c) les espèces régulières, qui sont celles dont la présence, de caractère généralement saisonnier, correspond soit à des apports de larves (passivement transportées par des courants, généralement engendrés par certains régimes de vents), soit à des migrations actives commandées par des facteurs d'ordre génésique ou trophique.

L'abondance des facteurs de pollution dans les estuaires est suffisamment évidente pour qu'il soit inutile d'en traiter ici. Tous les fleuves étant plus ou moins devenus de véritables collecteurs des eaux usées domestiques et industrielles de leurs bassins versants respectifs, il y a une large gamme de polluants que l'on trouve en estuaire et qui y subissent des modifications de leurs propriétés phym siques (précipitation, adsorption, désorption, etc.) modifiant leur distribution, ou des changements d'ordre chimique; ces polluants peuvent agir sur les êtres vivants. La connaissance de cette évolution physico-chimique est actuel. lement très insuffisante, presque autant que celle des seuils de toxicité aiguë et surtout chronique. A cette pollution apportée par le fleuve, s'ajoute évidemment celle liée aux implantations urbaines et industrielles, souvent très importantes dans le cas de fleuves navigables, des rives de l'estuaire luimême. D'une façon générale, on peut considérer qu'un estuaire est une zone où l'impact de la pollution est exacerbé par rapport au milieu fluvial et au milieu proprement marin.

Les espèces, que j'ai appelées précédemment permanentes, sont en général de faible intérêt économique, à l'exception toutefois de divers Bivalves (moules, huîtres, palourdes) qui y trouvent, grâce aux gradients de $\mathrm{T}^{\circ}$ et $\mathrm{S} \%$, une gamme de conditions favorables aux diverses étapes du processus de reproduction et, du fait de l'eutrophisation, des conditions de nourriture satisfaisantes. Bien entendu, la présence d'hydrocarbures peut rendre ces divers mollusques inconsommables, tout comme dans les milieux côtiers, et la concentration par eux de certaines substances organiques (Polychlorobiphényles en particulier) ou minérales (cuivre notamment) peut présenter des dangers pour la santé publique; bien entendu aussi, la production même des larves peut être affectée par l'action de nombreux polluants, auxquels les larves sont, en général, plus sensibles que les adultes.

Beaucoup plus importantes au plan économique sont les espèces que j'ai classées dans les régulières et qui présentent toutes le même schéma migratoire : la reproduction a lieu en mer, à plus ou moins grande distance du rivage, mais les larves ou les jeunes font une migration trophique vers les eaux saumâtres (estuaires ou lagunes). C'est le cas de nombreuses crevettes, principalement de la 
famille des Pénéides, et aussi de nombreux poissons, parmi lesquels les Mugilidés (muges ou mulets), les bars, les daurades, représentés sur de nombreuses côtes en zone tempérée et tropicale par des espèces différentes mais de comportement migratoire pratiquement identique. On pourrait y ajouter le «Milkfish» (Chanos) du Sud-Est asiatique, le pompano (Trachinotus) d'Amérique Centrale, et bien d'autres encore.

La relative fragilité des individus jeunes qui fréquentent les estuaires les expose évidemment à l'action des polluants chimiques, et la raréfaction ou la disparition dans certains estuaires (ou lagunes) de certaines de ces espèces, dites régulières, s'explique sans doute, en grande partie, plus par l'extinction des juvéniles que par des entraves à la reproduction des adultes. Il est vraisemblable aussi que la pollution thermique peut jouer un certain rôle; ce facteur, sauf lorsqu'il prend une intensité extrême, est plutôt bénéfique, puisqu'il accélère la crois- sance; néanmoins, on doit aussi tenir compte du fait que les larves ou les individus très jeunes, arrivant de la mer en estuaire, doivent réaliser leur équilibre ionique avec le nouveau milieu, ce qui implique une certaine dépense d'énergie; le fait que la température, en accroissant l'intensité du métabolisme, entraîne aussi un surcroît de demande d'énergie, peut être néfaste.

Ainsi, la composition, la distribution, l'abondance du peuplement des estuaires apparaissent-elles comme essentiellement conditionnées par l'intensité, la durée et la fréquence des agressions du milieu ambiant, agressions contre lesquelles, au fil des temps, la sélection a armé les espèces adaptées à ce milieu, aux plans de la morphologie, de la physiologie, du comportement. Les agressions nouvelles issues des activités de l'homme risquent, si l'on n'y prend garde, de contribuer à la destruction de ces écosystèmes complexes, mais dont la productivité est loin d'être négligeable.

\section{Discussion}

Président : M. A. BRIN

M. le Président remercie M. le Professeur PÉRÈs pour son passionnant exposé et ouvre la discussion.

M. KHalanski (Etudes et Recherches E.D.F., Chatou) intervient en ces termes :

Il a été signalé, dans votre exposé, que l'échauffement de l'eau accroit la pollution. L'influence du réchauffement sur la nocivité de nombreux polluants ne s'exerce toutefois pas toujours dans le sens d'un renforcement de la toxicité pour les organismes aquatiques. Une élévation de température peut, soit agir en synergie avec un toxique en favorisant son absorption, soit avoir une action antagoniste en augmentant la cinétique de l'excrétion de ce produit.

$\mathrm{Ce}$ que vous dites est partiellement exact, répond $\mathrm{M}$. le Professeur PÉRÈs; si on laisse de côté l'action de la température sur les pullulations anormales d'algues unicellulaires, ce que vous dites est exact, mais seulement pour certaines espèces et cela dépend de la température et aussi de l'importance du rejet.

La pollution thermique agit d'une façon très différente sur les diverses espèces et, comme on l'étudie essentiellement dans les mers tempérées, l'idée qu'on en a est, au fond, une idée très fausse, car dans une mer tempérée - prenons par exemple les côtes de l'ouest de la France - le peuplement d'une zone cotière et, par conséquent, (puisque sa dominante est marine) le peuplement d'une zone d'estuaire, comprend trois compartiments géographiques : il y a des espèces de caractère boréal, qui sont au voisinage de la limite sud de leur extension géographique; il y a des espèces atlanto-méditerranéennes, les « lusitaniennes », qui sont au voisinage de la limite nord; puis il y a les espèces tempérées. Il est certain que, s'il y a un réchauffement thermique en un endroit, les espèces boréales ou boréo-arctiques ne vont pas résister; au contraire, cela ne fera pas de mal aux espèces Iusitaniennes; cela leur fera même du bien et, comme elles étaient à ce moment au voìsinage de leur température limite froide, s'il y a une pollution à ce moment-là, elles se trouveront dans des conditions beaucoup plus favorables pour résister.

C'est un point dont on a très peu parlé dans les travaux sur la pollution thermique : on n'insiste pas assez sur le fait que, comme on l'a toujours étudiée dans les mers tempérées, on a affaire à un peuplement composite. Et, bien sûr, les réactions aux autres agressions : agression de salinité, agression de polluants, etc., varient suivant qu'il s'agit d'une espèce qui se trouve bien chez elle ou qui est au voisinage de sa limite supérienre ou de sa limite inférieure de tolérance.
Dans l'exposé de $M$. le Professeur Pérès, remarque M. Maquet. deux paramètres importants de l'écologie des estuaires sont considérés : le gradient de température et le gradient de salinité ; le gradient de turbidité n'a pas été cité. Il souhaiterait savoir si ce paramètre est considéré comme n'ayant pas d'influence sur l'écologie.

Le gradient de turbidité. précise M. le Professeur Pérès. a beancoup d'importance, surtout dans une zone de dilution, car il conditionne la position respective - liée à la turbidité d'ailleurs - d'une part, de la couche au-delà de laquelle la quantité d'énergie lumineuse n'est plus suffisante pour donner aux végétaux un bilan de photosynthèse positif (c'est ce qu'on appelle la profondeur de compensation) et, d'autre part, la pycnocline. Parfois, les eaux sont tellement troubles qu'il $y$ a une zone de production végétale limitée à une mince pellicule; si la pycnocline est beaucoup plus bas, cela n'a pas grande importance; mais, quand les eaux ne sont pas très troubles et que la pycnocline est au-dessus de la profondeur de compensation, il y a certainement une forte diminution de la production. Dans l'étang de Berre, par exemple, où la pycnocline se tient vers $6 \mathrm{~m}$, il y a des moments où, quand les eaux sont très troubles, la profondeur de compensation est au-dessus de la pycnocline; dans ce cas-là, une partie de la couche dans laquelle il pourrait $y$ avoir de la production est inutilisable et inutilisée.

M. Martin s'interroge sur la validité du schéma général de comportement des êtres vivants, exposé par M. le Professeur Pérìs, dans le cas des estuaires à marées et des côtes atlantiques; l'importance de la turbidité et du bouchon vaseux semble devoir modifier les processus observés, par exemple en Méditerranée.

Te pense, répond $M$. le Professeur Pérès, que les estuaires à marée se polluent moins que les autres, mais tout dépend de l'estuaire... Dans mon exposé, je n'ai pu donner que des généralités, mais chaque estuaire doit faire l'objet d'une étude particulière, sans idée préconçue, et en ntilisant le bagage de connaissances fondamentales - encore assez léger - dont nous disposons.

Mais je considère qu'il y a une différence fondamentale, du point de vue du peuplement, entre les estuaires des mers à marée ct les estuaires des mers sans marée. Les premiers ont toute une série de caractères communs, et notamment une plus grande facilité d'élimination des polluants, et le va-et-vient des masses d'eau est de nature à faciliter l'établissement de peuplement normaux. 
M. KHalanskr intervient ensuite en ces termes:

$1^{\circ}$ On attribue généralement la prolifération du phytoplancton, qui se traduit par des «eaux décolorées», à quatre causes : I'eutrophisation, la dessalure, une température élevée et l'isolement d'une masse d'eau dans laquelle se produit une explosion de population. Les trois derniers facteurs sont liés, la stabilité dépend de la salinité et de la température. Connait-on actuellement l'importance relative de ces facteurs dans le déclenchement des eaux colorées?

$2^{\circ}$ L'infuence de la température est d'autant plus difficile à préciser que tous les facteurs ne sont pas connus. Il est possible que l'apparition d'eaux décolorées en été corresponde à un épuisement des réserves nutritives ou à une modification du rapport $N / P$, facteurs qui interviennent précisément pendant la saison chaude. On peut penser d'autre part que, dans l'exemple que vous avez cité, la dessalure ne représente pas le seul facteur aui détermine le phénomène puisque Blanc et Leveau ont mis en évidence une concentration planctonique deux fois plus forte au débouché des eaux du Rhône, en mer libre, que dans le golfe de Fos, soumis à une dessalure comparable.

M. 1e Professeur PÉRÈs répond :

C'est-là une question très pertinente et je vais vous faire une réponse qui va beaucoup vous décevoir. Je vous ai parlé de ces facteurs de pullulation anormale, excessive, que sont les facteurs simples; en réalité, il y en a vraisemblablement un autre, ou d'autres... Depuis environ trente ans, des efforts presque désespérés sont faits pour comprendre l'origine de ces multiplications anormales d'algues souvent toxiques, car c'est une condition presque sine qua non de toute une série d'opérations d'aquaculture en zone tropicale : tant qu'on ne pourra pas maitriser ces pullulations anarchiques, on ne pourra jamais élever des huitres et des moules dans certaines aires côtières on lagunaires situées en zone intertropicale. Le seul progrès sensible qu'on ait fait, à mon avis, au cours des dernières années, c'est la mise en lumière de l'importance du rapport $N / P$. Il y a vraisemblablement aussi une histoire de facteur de croissance, probablement liée à l'entraînement, par exemple, de vitamines B 12 par les eaux de ruissellement. Pour l'instant, malgré ces efforts - et on en fait beaucoup dans mon laboratoire - l'explication de ces poussées anarchiques reste encore à trouver. Que ceia succède à des périodes de grand drainage, c'est certain; qu'il soit nécessaire que la température soit élevée, c'est certain; on ne verra jamais un phénomène de pullulation anormale en mer du Nord au mois de janvier, ou sur les côtes de Bretagne dans le même mois. La salinité n'a probablement qu'un rôle très indirect. Le milieu fermé est certainement très important dans la valeur atteinte par le rapport $N / P$; il y a aussi le facteur de croissance de petites molécules organiques, l'entraînement de vitamines du groupe B 12 , de biotine, etc.

J'en viens maintenant aux conditions de milieu dans le golfe de Fos; c'était, il y a trois ans, une zone relativement saine, sauf au point de vue de certains polluants chimiques qui étaient apportés par le contre-courant du Rhône. L'eutrophisation est en train de progresser. Je crois que le problème des facteurs organiques reste posé. Quand à la température, je ne suis pas tellement de votre avis : je pense que le réchauffement anormal est certainement un ćlément qui déclenche le phénomène. Vous dites que c'est une conséquence de l'eutrophisation; pas forcément, parce que, dans les baies tropicales ou les lagunes côtières, comme la lagune Rodrigo à Rio-de-Janeiro, où ces phénomènes interviennent avec une périodicité que l'on n'a jamais réussi à déterminer, il fait toujours chaud et il y a toujours une quasi-saturation de sels minéraux nutritifs.

Personne ne demandant plus la parole, $M$. le Président remercie M. le Professeur PÉRÈs et tous ceux qui ont animé la discussion il donne la parole à M. Gauthrer pour l'exposé de la communication qu'il a rédigé avec MM. AUBERT et J. AUBERT. 